\title{
CORRIGENDUM
}

\section{Mechanism and relevance of EWS/FLI-mediated transcriptional repression in Ewing sarcoma}

\author{
S Sankar, R Bell, B Stephens, R Zhuo, S Sharma, DJ Bearss and SL Lessnick
}

Oncogene (2016) 35, 6155-6156; doi:10.1038/onc.2016.142; published online 27 June 2016

Correction to: Oncogene (2012) 32, 5089-5100; doi: 10.1038/onc. 2012.525; published online 26 November 2012

The authors were recently made aware of concerns regarding discontinuities and duplications of portions of Figures $5 \mathrm{e}, 5 \mathrm{f}$ and $6 \mathrm{~b}$ of this publication. We sincerely apologize for lack of clarity and errors involving these figures. To resolve these issues, we provide the following discussion and updated figures.

Duplications: The experiments shown in Figures $5 e, 5 f$ and $6 \mathrm{~b}$ were performed as one large co-immunoprecipitation experiment in which the FLAG antibody was used to immunoprecipitate FLAGtagged wild-type EWS/FLI, $\Delta 22$ and mutant 9. Portions of the same immunoprecipitation were then analyzed on separate western blots for FLAG, HDACs, CHD4 and MTA2. Because the FLAG western blot served as the control for each of the other analyzed proteins, the same control FLAG western blot was shown in each of the aforementioned figures. This was an intended duplication, but we should have explicitly stated this in the manuscript.
Discontinuities: In Figure 5e, a discontinuity was noted in the FLAG western blot panel. This discontinuity was a result from the removal of western blot lanes that were not included in the figure. We now provide an updated Figure $5 e$ in which the FLAG western blot sections are clearly separated to demonstrate that they were discontinuous portions from the same western blot.

In Figures $5 \mathrm{f}$ and $6 \mathrm{e}$, discontinuities were also noted in the duplicated FLAG panels of the wild-type EWS/FLI immunoprecipitation lanes. This panel was altered to remove degradation products in that lane. We now provide updated figures in which these elements remain unaltered. The updated figure is taken from a different western blot exposure that still has hand-drawn molecular weight markings in the middle lane.

The updated figures do not change the interpretation of the data or the stated conclusions in the original Oncogene manuscript.

The authors apologise for any inconvenience caused by this error.

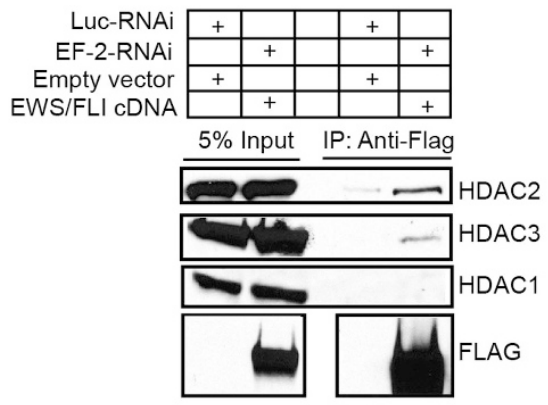

f

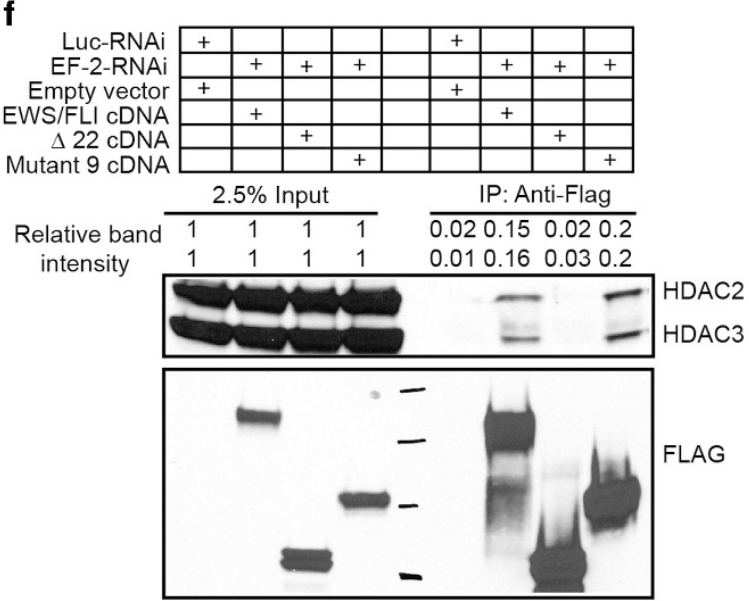

Figure 5. 
b
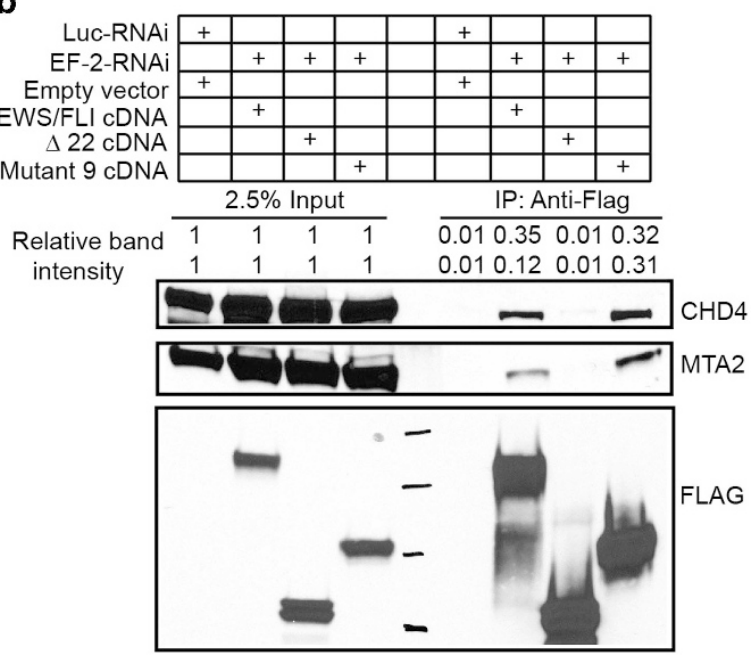

Figure 6. 